\title{
PERCEPCIONES Y RECONOCIMIENTO LOCAL DE FAUNA SILVESTRE, MUNICIPIO DE ALCALÁ, DEPARTAMENTO DEL VALLE DEL CAUCA, COLOMBIA
}

\author{
Narli Johana Aldana Mejía ${ }^{1}$ \\ Mónica Díaz Porres ${ }^{2}$ \\ Alexander Feijoo Martínez ${ }^{3}$ \\ Heimar Quintero ${ }^{4}$
}

Recibido el 19 de mayo de 2015, aprobado el 7 de julio de 2015 y actualizado el 16 de mayo de 2016

DOI: 10.17151/luaz.2016.43.4

\section{RESUMEN}

En los Andes centrales de Colombia se documentaron las percepciones y reconocimiento local de aves, mamíferos, anfibios y reptiles. Se entrevistó a campesinos con edad superior a 30 años para situar áreas de hábitat y reconocer especies en usos y coberturas del terreno. El reconocimiento de los agricultores se tradujo por los investigadores-mediadores en la formulación de categorías taxonómicas con visitas a los predios, avistamiento y la ayuda de guías de campo. Se reconocieron 168 especies: 103 de aves, 31 de mamíferos, 17 de anfibios y 17 de reptiles. Cuatro Análisis de Componentes Principales relacionaron la mayor competencia de campesinos para reconocer y percibir aves, mamíferos, anfibios y reptiles con diferencias significativas en los usos del terreno (valor $p<0,01$, prueba Monte Carlo); se separaron las especies de aves para uso directo y hábito alimentario; mamíferos para uso directo e indirecto; se agruparon los reptiles con valor de uso comercial, ornamental, artesanal y cacería. Las aves fueron las de mayor presencia en los usos y coberturas del terreno (frutales, 47,5\%; platanal/bananal, 45\%; cafetales con sombrío, 40\%). Se confirmó la necesidad del encuentro con los repertorios culturales locales para potenciar la política pública de conservación de la diversidad biológica y la asociación de la calidad de los hábitats para la fauna silvestre con los estilos de agricultura de la localidad.

\section{PALABRAS CLAVE}

Conocimiento local, etnozoología, usos terreno, valor uso, fauna silvestre. 


\title{
WILDLIFE PERCEPTION AND RECOGNITION IN THE MUNICIPALITY OF ALCALÁ, DEPARTMENT DEL VALLE DEL CAUCA, COLOMBIA
}

\begin{abstract}
To document the perception and recognition of local bird, mammal, amphibian, and reptile species, located in the Central Andes of Colombia were selected. Farmers aged 30 years and older were interviewed to identify areas of habitat, species, and land use coverage. The local recognition made by the local farmers was translated by researchers into taxa, complemented with visits to farms, sightseen, and with the help of field guides. Overall, 168 species were documented of which 103 corresponded to birds, 31 to mammals, 17 to amphibians, and 17 to reptiles. The results of four principal component analyses show that the local farmers are capable of recognizing and perception birds, mammals, amphibians, and reptiles, with significant differences in land uses ( $p<0.01$, Monte Carlo test). Bird species were grouped according to direct use and eating habits; mammals were grouped according to direct and indirect use; and finally reptiles were grouped according to use as commercial, ornamental, artisanal, and hunting. Birds ranked highest in presence in all land uses and such land covers (fruit trees, 47.5\%; plantain and banana plantations, 45\%; shade-grown coffee, 40\%). The needs for meetings and the acquisition of local cultural repertoires to improve governmental policies regarding biodiversity conservation and to relate wildlife habitat quality with local farming systems was confirmed.
\end{abstract}

\section{KEY WORDS}

Local knowledge, ethnozoology, land use, use value, wildlife.

\section{INTRODUCCIÓN}

Los efectos deliberados-involuntarios de la ocupación humana del territorio hacen emerger hábitats de diferente calidad para otras formas de vida según la intensidad y frecuencia de la perturbación (Hobbs et al., 2006; Marshall, West y Kleijn, 2006). Cuando el paisaje resultante se estructura como mosaico en el que alternan parches de vegetación secundaria con parcelas de cultivo, ofrece a la vida silvestre hábitats para refugio, nidificación y creciente oferta de alimento (González, 2003a). 
En el municipio de Alcalá (Valle del Cauca, Colombia) durante la última década del siglo XX se incrementó la superficie ocupada por los cultivos en surco continuo y a plena exposición solar de café variedad Colombia, disminuyeron los montes maduros (selva andina secundaria) y solo quedaron relictos de selva, parches de guadua en orillas de quebradas. Entre 1995 y 2002, la crisis fitosanitaria y de mercado obligaron a los empresarios a reducir los cafetales y aumentar los monocultivos como maíz y fríjol, plátano y el pastizal (Cynodon nlemfuensis) para cría de bovinos (Zúñiga, Feijoo y Quintero, 2003, 2004). Algunos agricultores conservaron el cafetal con sombra asociado con yuca, plátano, frutales y cítricos y en ocasiones aisladas con árboles maderables (nogal cafetero y laurel), y también conservaron relictos de vegetación secundaria a orillas de las quebradas (Zúñiga et al., 2003, 2004; Feijooet al., 2007; Feijoo et al., 2011). En la actualidad el paisaje rural del municipio se caracteriza por combinar cafetales con sombrío o a libre exposición solar, platanales, pastizales o mezclas de ellos, con pequeños parches de vegetación secundaria a orillas de corrientes hídricas. Aunque estos espacios pueden generar lugares hostiles o amigables para la biodiversidad (RuizCobo, Feijoo y Rodríguez, 2010; Feijoo et al., 2010), no se ha construido la información que diferencie los refugios propicios o hábitats más amigables para la conservación de la biodiversidad faunística, resultantes como efecto no buscado de la colonización de los Andes del centro de Colombia.

El enfoque de conservación de la biodiversidad como responsabilidad nacional y supranacional comienza a ser complementado por la perspectiva de que el manejo de la diversidad biológica representa una oportunidad para comprender las necesidades, prioridades, percepciones, sistemas de conocimiento locales y para alentar el compartir del diálogo de saberes (Sheil et al., 2006). La valoración de los conocimientos, percepciones y prácticas de los agricultores es esencial para desarrollar estrategias de manejo local que incluyan las aspiraciones de ellos (Nyeko et al., 2002).

Para abordar los interrogantes o las estrategias para construir la comunicación entre la dupla investigador-campesino, se precisa considerar la colaboración de los actores locales en la identificación, ecología, conservación, valoración y usos de la biodiversidad (Sheil y Lawrence, 2004), así como consolidar medios de evaluación que mejoren la gestión local de los recursos naturales y la conservación (Charnley, Paige y Jones, 2007; Millington et al., 2010) y contribuir con programas de educación ambiental que incorporen la etnozoología (Zúñiga et al., 2013). 
Las aves, mamíferos, anfibios y reptiles por su visibilidad macroscópica tienen representación simbólica en los repertorios culturales de las localidades, esto es, en costumbres, restricciones sociorreligiosas, en mitos y cosmovisiones. Los agricultores valoran las especies de acuerdo con características, funciones, el manejo de los recursos (Millington et al., 2010) y las formas culturales de interactuar dentro del medio. Las culturas humanas, los sistemas de conocimiento, religiones, valores patrimoniales, interacciones sociales y servicios, siempre han sido influenciadas y moldeadas por las condiciones de los ecosistemas en los que se basa la cultura (MEA, 2005a, 2005b). Al mismo tiempo, la gente siempre ha ayudado a configurar el entorno para mejorar la disponibilidad y valoración de los recursos (Tengberg et al., 2012; Zúñiga et al., 2013), hechos que se asocian con el proceso de aprendizaje, que producen conocimiento o experiencia sobre el entorno, y que se denomina percepción, la cual depende del actuar del individuo en su contexto (Durand, 2008), permite la comprensión mutua y forma valores (Tuan, 1974), se desarrolla conocimiento (De Albuquerque y De Albuquerque, 2005), cambia nuestra realidad (Zúñiga et al., 2013), y lo que percibimos es función directa de la forma en que actuamos y, por lo tanto, el conocimiento obtenido a través de la percepción directa es lo práctico, es el conocimiento de lo que ofrece el ambiente para la prosecución de la acción en la que el perceptor se involucra (Ingold, 2000).

A partir de las consideraciones anteriores se formuló la siguiente hipótesis:

-El conocimiento de la riqueza de especies de la macrodiversidad de animales vertebrados en los Andes del centro de Colombia se puede reconocer, valorar y usar mediante la estrategia de documentar las percepciones de los actores de las localidades.

En el trabajo se propuso como objetivos: documentar las percepciones y el reconocimiento de aves, mamíferos, anfibios y reptiles en el repertorio cultural de la localidad, y diferenciar los usos del terreno como amigables y hostiles para la vida silvestre según la intensidad de uso del terreno. 


\section{MATERIALES Y MÉTODOS}

\section{Área de estudio}

El área de estudio se localizó en el municipio de Alcalá en Colombia (4²43'18,25" Norte, 7551'22,91" Oeste y 4º38'56,85" Norte, 75²42'11,94" Oeste) situado en la vertiente occidental de la Cordillera Central de los Andes (Figura 1). El trabajo de campo se llevó a cabo entre diciembre de 2004 y agosto de 2005. El clima de la región se caracteriza por dos temporadas secas (diciembre-febrero y junio-agosto) y dos de lluvias (marzo-mayo y septiembre-noviembre). El área varía en altitud (900-2000 msnm), precipitación anual $(1350-2400 \mathrm{~mm})$, temperatura $\left(18-24^{\circ} \mathrm{C}\right)$ y humedad relativa (65-80\%). En el estilo de agricultura campesina se combinan el uso de prácticas tradicionales y producción diversificada con introducción de cultivos semestrales, semipermanentes y cría de animales que abastecen la subsistencia de las familias; en las fincas se destina parte del terreno para favorecer el crecimiento de vegetación secundaria y conservar las márgenes de las quebradas y ríos con vegetación típica de las áreas. En fincas empresariales se han introducido técnicas intensivas y uso de insumos de síntesis química, o sembraron el terreno en los últimos 15 años con pastizales o cultivan variedades mejoradas de café, yuca, plátano o combinaciones de cultivos en surco (Feijoo et al., 2011).

En el paisaje se situaron seis ventanas al azar de $1 \mathrm{~km} 2$ de cobertura con 16 puntos georreferenciados cada $200 \mathrm{~m}$ (Feijoo et al., 2011), que permitieron la selección de 51 fincas en nueve veredas, en las que se realizaron entrevistas a campesinos de sexo masculino, con edad superior a los 30 años (Tabla 1). Para realizar el trabajo detallado se escogieron 18 predios de propietarios 0 administradores con base en el tiempo de permanencia en la finca, el saber relacionado con la fauna silvestre y el tamaño de muestra (Daniel, 1996) estimada con las fórmulas:

$$
\text { no }=z^{2} \frac{P * Q}{d^{2}}
$$

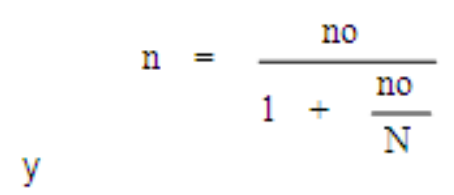

donde: no = tamaño de la muestra sin ajustar $(27,23) ; \mathrm{n}=$ tamaño de la muestra efectiva $(17,75=18) ; \mathrm{N}=$ población total $(51) ; \mathrm{P}=$ probabilidad de ocurrencia del 
evento (50\%); Q = probabilidad de no ocurrencia del evento (1-P) (50\%); z = nivel de confianza del estudio $(88,5 \% Z=1,20036)$; $d$ = porcentaje de error $(11,5 \%)$.

Las fincas se visitaron cada ocho o 15 días (15 a 20 visitas, durante nueve meses), de acuerdo con la disponibilidad de tiempo de los agricultores y se recopiló la información con métodos cualitativos (Deslauriers, 1991) con énfasis etnográfico (Guber, 1994). El medio de acercamiento y recolección de datos fue la entrevista semiestructurada que permitió indagar acerca del conocimiento local, las percepciones y las actitudes de los agricultores con relación al ambiente, situar áreas de hábitat y reconocer las especies en los usos y coberturas del terreno de las fincas (cacaotal, cafetal con sombrío, platanal/bananal, yucal, maizal, caña panelera, frutales, pastizal; relicto de vegetación secundaria; cuerpos de agua tales como quebradas, nacimientos y humedales; guaduales). El intercambio se estableció a partir de la construcción de lazos afectivos entre las personas, el lugar y el ambiente en el que moran, para determinar por medio de las percepciones, las características y registros de los espacios para agricultura y lugares de afluencia de la fauna silvestre. 


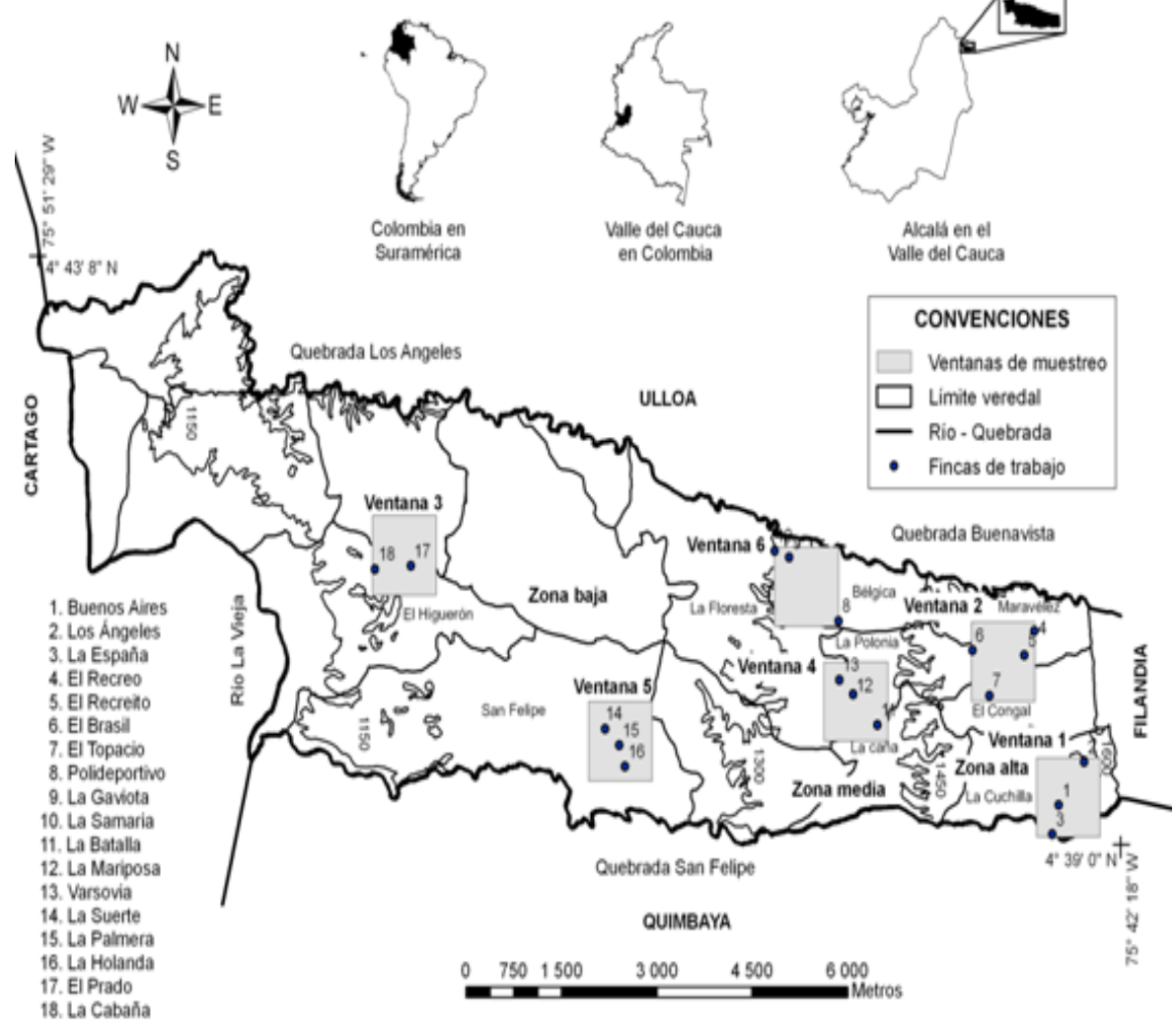

Fuente: los autores

Figura 1. Localización de las fincas en el área de estudio.

Tabla 1. Caracteristicas de los agricultores y de las fincas en el estudio en el municipio de Alcalä

\begin{tabular}{|c|c|c|c|c|c|c|}
\hline Vetedia & Fines & $\begin{array}{l}\text { Edad } \\
\text { (años) }\end{array}$ & $\begin{array}{c}\text { Nivel } \\
\text { Escolar }\end{array}$ & Finex & $\begin{array}{l}\text { Ares } \\
\text { (has) }\end{array}$ & Ceberturas /Usos del Suelo \\
\hline Lo Cuenis & ${ }^{*}$ & 45 & Jopnmes & Buthos Aires & 8.8 & 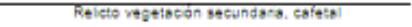 \\
\hline Ls Cuensa & $2 *$ & 52 & 20phasts & Los $\operatorname{sag} \theta+4$ & 6.08 & 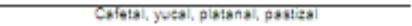 \\
\hline $7 \operatorname{mos}$ & $3^{+}$ & 65 & 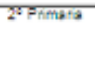 & LEEGSERT & 6 & 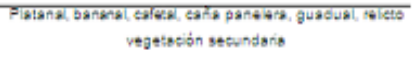 \\
\hline Maravobz & $4^{+12}$ & 52 & 3:7nmara & ElRocero & 8,98 & 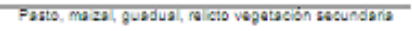 \\
\hline Maraviaz & $\sigma^{2}$ & 88 & $30=\pi m a r a$ & E Roovito & 7,1 & 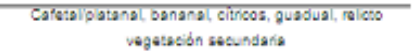 \\
\hline Maravoz & $8^{2}$ & 50 & 207mara & 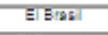 & 236 & Fastzal, Gokatal, phianal, guasual \\
\hline ECongal & $7^{2}$ & 84 & Eachilar & EITOpaco & 12,16 & 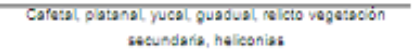 \\
\hline E Hquerón & $17^{*}$ & 72 & $2^{0}$ Prmara & ElPraso & 13.1 & 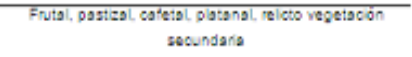 \\
\hline El Haverion & $16=$ & 32 & 40prmarg & Lo Casonto & 23.04 & Posiesl gusouge plotsmbl \\
\hline Lo $\cos \theta$ & $11^{*}$ & 48 & 5rpamers & Lo 685618 & 28 & 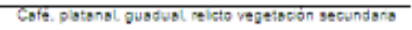 \\
\hline Do prisha & $12=$ & 30 & $7+\operatorname{seshitit}$ & $601.16 \times 9093$ & 15.35 & 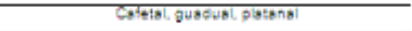 \\
\hline Letrobna & $13{ }^{\prime \prime}$ & 32 & 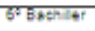 & Vansovis & 48 & 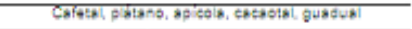 \\
\hline 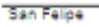 & $14^{\circ}$ & 69 & $2 \cdot 7 \operatorname{mmb}$ & LS 30640 & 65 & 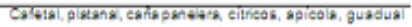 \\
\hline $\operatorname{sen} F=04$ & $15^{*}$ & 45 & Exchist & 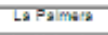 & 1822 & Cosial noleshas \\
\hline $\operatorname{san} F_{0} 00$ & $16^{2}$ & 49 & g:Fimara & La hasanos & 17,32 & Pancal guadus, patanal \\
\hline 36962 & $8^{4+}$ & 82 & $2^{0}=$ rimara & Poldoportivo & - & Fastiza, calotal \\
\hline 86962 & $3^{* 4}$ & 48 & $\delta^{2}$ sachler & Le Geviota & 8.56 & Citroos, cofote, pletane, guesual \\
\hline Edgles & $10^{\circ}$ & 70 & 20 prmara & La Samana & 14.08 & Patangl catetol ma zal, gescicola \\
\hline
\end{tabular}

Haga clic sobre la imagen para ampliarla 
Se partió de preguntas básicas individuales: cuántas especies, cómo se diferencian, dónde se encuentran, qué características presentan, cómo valoran y usan la fauna silvestre. Las respuestas fueron evaluadas para reconfigurar las preguntas y construir nuevos interrogantes que ayudaran a comprender lo que se pretendía y cómo el agricultor modifica el sentido de la investigación. Posteriormente se usó la redundancia en las preguntas cómo método que permitiera entender el significado, reconocer cómo lo percibimos, cómo se estructura, valora y usa desde lazos afectivos entre las personas (agricultores y expertos), para identificar en el conocimiento específico, las imágenes y las actitudes que una persona adquiere de su relación entre el entorno y las cosmovisiones (Zúñiga et al., 2013).

Los 18 agricultores se reunieron en tres talleres comunitarios para confirmar la presencia de aves, mamíferos, anfibios y reptiles en los diversos usos y coberturas del terreno. Se utilizó el Método de Distribución de Piedritas (MDP) (Sheil et al., 2003) para determinar la importancia de los mismos en las fincas como hábitats para la biodiversidad, afianzar y refrendar los registros obtenidos en las entrevistas individuales. En el mismo se confirmó la distribución de 1 a 100\% de aves, mamíferos, anfibios y reptiles reportados por los campesinos para cada uso del terreno. Estos se representaron con tarjetas ilustradas, dibujos de mamíferos, aves, reptiles y anfibios, las cuales se asignaron a tres grupos de seis personas, para establecer las asociaciones de acuerdo con la presencia/ausencia de animales. El reconocimiento local de los organismos por los agricultores se tradujo por los investigadores-mediadores en categorías taxonómicas (Reino, Phylum, Orden, Familia, Género y Especie) con la ayuda de guías de campo para aves, mamíferos, anfibios y reptiles y un listado de 190 especies distribuidas entre 900 y 1.600 msnm en el Eje Cafetero colombiano (Hilty y Brown 1986; Emmons, 1991; Caicedo, 1994).

La información de las entrevistas individuales, los talleres para reafirmar el conocimiento de los agricultores y las observaciones de los expertos en campo, se ordenaron en bases de datos de doble entrada con registros cuantitativos de presencia/ausencia de la especie, avistamientos en fincas y, además, registros organizados de acuerdo con dos categorías: a) uso directo: consumo (alimento, medicina), producción (artesanal, ornamental, comercio), científico (investigación), recreacional (cacería, observación, turismo); y b) indirecto: cultural (mitos y rituales, arte y literatura, pedagógico, negativo) y benéfico (McNeely et al., 1990; Tobasura, 1996; Swift, Izac y Noordwijk, 2004; MEA, 2005a, 2005b). 
Se utilizó Análisis de Componentes Principales (ACP) debido a la procedencia multivariada de los datos provenientes del reconocimiento, distribución en las coberturas y usos del terreno, valoración y uso de aves, mamíferos, anfibios y reptiles, lo que posibilitó transformar el conjunto de variables cuantitativas o discretas en otro nuevo (componentes principales) con funciones lineales de los registros, ortogonales, independientes unas de otras. La variación total entre componentes principales fue igual a la variación total de los datos originales de manera que las diferencias entre ellas no se pierden en la transformación, y la varianza asociada con cada nueva variable se ordenó en forma decreciente (Vargas, 1984).

Para cumplir con el propósito se hicieron cuatro ACP: el (i) se realizó con los registros de aves, mamíferos, anfibios y reptiles, el (ii) para aves, el (iii) con mamíferos y el (iv) con reptiles. En cada uno de ellos se determinó el conocimiento local relacionado con la capacidad para diferenciar especies de acuerdo con el territorio que ocupan (fincas), la distribución e identificación de asociaciones entre usos y coberturas del terreno y valores de uso directo e indirecto. Además, se hizo análisis discriminante, mediante el uso de la prueba de permutación (Monte Carlo) para calcular la inercia total de las interclases para cada distribución al azar de las especies con los valores de uso, y medir la distancia matemática de las especies de animales y los valores de uso (Velásquez et al., 2005). Los análisis estadísticos se realizaron con el programa ADE4 (Thioulouse et al., 1997).

\section{RESULTADOS}

\section{Valoración y reconocimiento local de aves, mamíferos, anfibios y reptiles}

El trabajo conjunto entre investigadores y las comunidades locales arrojó el reconocimiento de 168 especies de fauna silvestre: 103 de aves pertenecientes a 16 ordenes (42 especies de Passeriformes, nueve Apodiformes, ocho Psittaciformes, siete Piciformes, seis Falconiformes, cinco Columbiformes, cuatro Accipitriformes, cuatro Caprimulgiformes, cuatro Cuculiformes, tres Tinamiformes, tres Strigiformes, dos Galliformes, dos Cathartiformes, dos Coraciiformes, una Charadriiformes, una Pelecaniformes). En mamíferos se identificaron 31 especies situadas 10 en Rodentia, seis en Chiroptera, cinco en Didelphimorphia, cinco en Carnívora, dos en Pilosa, y en Cingulata, Xenarthra y Lagomorpha, una especie 
respectivamente. En anfibios se reconocieron 17 especies pertenecientes al orden Anuros (16 ranas y un sapo). En reptiles hubo 17 especies, situadas ocho dentro del grupo de las serpientes (Squamata: Serpentes), seguido por siete lagartos (Squamata: Sauria) y dos tortugas (Testudinata) (Tabla 2).

Treinta especies de aves fueron las más reconocidas con distribución mayor o igual al $80 \%$ para los usos del terreno, entre las cuales se destacan: Coragyps atratus, Columbina talpacoti, Rupornis magnirostris, Megascops choliba, Tapera naevia y Momotus aequatorialis. Los mamíferos más distinguidos fueron: Dasypus novemcinctus y Sciurus granatensis (100\%); seguidos por Didelphis marsupialis (94,4\%) y Gloosophaga soricinay Mustela frenata $(88,9 \%)$. De las 17 especies de reptiles registradas, Chironius monticola(94,4\%), Micrurus mipartitus y Erythrolamprus bizona (88,9\% y $89 \%$ respectivamente) presentaron la mayor presencia.

Tabla 2. Reconocimiento de fauna silvestre por campesinos en el municipio de Alcala

\begin{tabular}{|c|c|c|c|c|c|}
\hline \multicolumn{6}{|c|}{ Aver } \\
\hline Nombre comùn & Nombre clentifico & Nombre común & Nombre clentifloo & Nombre común & Nombre Clentiflco \\
\hline Gavilan $\phi$ & Accipler Sylatus & Trepajor \& & xponornynchus gurra:us & Lora malcera: & monus tumutuosus \\
\hline Agulla $\$$ & bureo playprerus & Garrapatero U & Crovopnaga any & Turpla: · & $\begin{array}{l}\text { leserus crrysa:er } \\
\text { Mamproceus }\end{array}$ \\
\hline Rapina $\phi$ & caracara cnerway & Soledad U & Maja cajana & Toche : & $\begin{array}{l}\text { olmilatus } \\
\text { mampnoceus }\end{array}$ \\
\hline Buno $\Phi$ & Ciccasa wrgata & Cuco enano $\amalg$ & Maya minura & Toche & famimigerus \\
\hline Rapina $\phi$ & raco coumoarius & Rastrojerou & Heminoplex orunnescens & Careperro. & sataror sylabectus \\
\hline Rapina $\Phi$ & racorungularis & Piscuiz山 & synalaus avescens & Soldadio & sturnela mowar/s \\
\hline Rapina $\phi$ & racospanerus & Tute 4 & synalaus azarae & Azulepo. & Iraupls eplscopus \\
\hline Rrapina $\phi$ & kona punnoes & Tute 4 & synalavus unuruta & Azule,o tumadio. & Inraypls paimarun \\
\hline Currucuto $\Phi$ & hegascops chonloa & $\begin{array}{l}\text { Tres ples } \amalg \\
\text { Bataras }\end{array}$ & $\begin{array}{l}\text { lapera naeva } \\
\text { Inaminopnuss }\end{array}$ & Mirla. & lurous ocsoletus \\
\hline Halcon pajarero $\phi$ & Macrastyr funcoms & carca,jaja U & multsstratus & Cnicagua. & lurous ignoows \\
\hline Plgua $\Phi$ & Lawvago cnimacnima & Cucarachero 4 & lrogocyyes asoon & Tomine:o- & Aาiz แa savcerrore \\
\hline Gavilan $\Phi$ & rupornis magnurosyis & & & Tomine:po- & Amazma rzaca: \\
\hline Lechuza $\Phi$ & lyco ava & Sacanero colujo $\Delta$ & Enoerizouses nerovola & Milelero - & coereoa naveola \\
\hline Guala 4 & canartes aura & Canario $\Delta$ & sucals naveola & collorl- & $\begin{array}{l}\text { crayoura oumonv } \\
\text { cinorostuoon }\end{array}$ \\
\hline Ga nazo $\psi$ & coragyps avanus & Sen ero $\Delta$ & sporopnia minura & Tomine:0- & mellsugus \\
\hline MonjtaK & ruvvcola pica & Sen & sporopnua nyprooms & Tomine:0- & coeugena coengena \\
\hline Atrapamoscas $K$ & Mylarchus tuosercuver & Satarin $\Delta$ & voavna jacarina & Tomine:0- & Lorvera nooviclas \\
\hline \multirow{2}{*}{$\begin{array}{l}\text { Rana venenosa del } \\
\text { Cauca }\end{array}$} & & & \multicolumn{3}{|l|}{ Eeurrococtyus } \\
\hline & $\begin{array}{l}\text { Uenorocases oomoeses } \\
\text { venorocates }\end{array}$ & & \multicolumn{2}{|l|}{ orpacobates } & Eevorrocactyus sp \\
\hline \multirow[t]{2}{*}{ Rana veายกางa } & histrionicus & & \multicolumn{3}{|l|}{ Eeuverocactyus paine? } \\
\hline & & & Reptlles & & \\
\hline Lagarto & Anous venyוmacuatus & Falsa coral & eryorolamprus ozona & Lagarto & roycrus gumurosus \\
\hline Jesueristo & basmscus gaeritus & Iguana & guana เguana & Camaleon & roycrus marnoratus \\
\hline Cazajora negra & Cuena cleia & Tapaculo & Kunos:ernon leveossornus & Granasila & spuotes punatus \\
\hline Jueteavora & Cnironves monocola & Guardacaminos & Lopns sp & Salamanqua.ja & Inecacactyus rapucayda \\
\hline Fasax & Lupsas sancyoanns & Mataganajo & sucrurus oumerm & Tortuga nicotea & Iracremys scripra \\
\hline Lagarto espinoso & ecnunosaura norrioa & Rado de $a_{1}$ & sucrurus mpartus & & \\
\hline
\end{tabular}

Haga clic sobre la imagen para ampliarla 
En el ACP (i) los dos primeros componentes representaron el $84 \%$ de la variabilidad total. El C1 $(68 \%)$ agrupó reptiles $(0,89)$, aves $(0,86)$ y mamíferos $(0,83)$, los que se relacionaron con la mayor competencia de los campesinos que viven en las fincas Buenos Aires, La Suerte, Polideportivo, La Gaviota y El Topacio para percibir especies de los tres grupos. En estos predios se distribuye el terreno entre diversos agroecosistemas tradicionales (cafetal con sombrío, platanal/bananal, frutales, relicto de vegetación secundaria y guaduales) mezclados con áreas en las que se permite el crecimiento de vegetación típica de sucesiones antropogénicas. El C2 (16\%) incluyó anfibios $(0,96)$, grupo que se asocia más con el reconocimiento de los agricultores que moran en La Mariposa, La Cabaña y Brasil (Figura 2), predios que se caracterizan por el predominio de sistemas de cultivo industrial (cafetal a libre exposición solar, pastizales) con alto uso de insumos de síntesis química y presencia de cuerpos de agua. Las diferencias fueron significativas para el reconocimiento de aves, mamíferos, anfibios y reptiles por los agricultores en los usos del terreno de las fincas (valor $p$ $<0,01$, prueba Monte Carlo).

Los primeros dos ejes del ACP para aves (ii) explicaron el $41,4 \%$ de la inercia (25,5\%, 15,9\%, respectivamente). El eje 1 separó los valores de uso directo usados con fines comercial $(0,90)$, ornamental $(0,88)$ y cacería $(0,74)$, que corresponden con aves frugívora-insectívoras, granívoras y frugívoras tales como Thraupis episcopus, Forpus conspicillatusy Aratinga wagleri; mientras que el eje 2 aglutinó las especies que se valoran por el uso artístico y literario $(0,76)$, alimento $(0,60)$ y medicina $(0,58)$, que atañen con hábitos alimentarios carroñero y omnívoro, entre las que se destacan Coragyps atratus yCrypturellus soui (Tabla 3). Relacionado con el C2, se encontró que algunas especies de aves son usadas para la alimentación (Crypturellus obsoletus, Momotus aequatorialis), el comercio (loras, Pionus spp., Amazona amazonica) y en ornamento (Thraupis episcopus, Icterus chrysater).

En el ACP (iii) dos componentes explicaron el $71,4 \%$ de la variabilidad total. El C1 $(55,5 \%)$ agrupó los mamíferos con uso directo e indirecto para beneficio $(0,92)$, investigación $(0,91)$, medicinal $(0,89)$ y mitos y rituales $(0,89)$ (Tabla 3). Las especies Dasypus novemcinctus yCabassous centralis fueron las especies de mayor uso con fines medicinales, especialmente por el uso de la sangre como cura para el asma. Los murciélagos Desmodus rotundus, Myotis riparius, Gloosophaga soricina y los ratones Akodon urichi, Hetotorys desmarestianus y Oryzomys capito se percibieron como negativos porque afectan a la ganadería bovina o a los cultivos respectivamente. El C2 (15,9\%) se relacionó con la 
observación $(0,62)$, ornamento $(0,60)$, y alimento $(0,52)$, y se denominó como usos para la satisfacción personal. En la asociación con el componente, se comprobó que los mamíferos son los de mayor uso de carne para alimentación (Sylvilagus brasiliensis y Dasyprocta spp., Didelphis marsupialis y Dasypus novemcinctus), o también se extraen la piel, patas y uñas para elaborar artesanías, producir ungüentos (Didelphis marsupiales, Metachirus nudicaudatus) o tapetes (Sylvilagus brasiliensis).

Siete especies de reptiles se relacionaron como fuente de alimento, artesanal, ornamental, cacería, observación, negativo (Trachemys scripta, Iguana iguana, Cleia cleia, Erythrolamprus bizona, Spilotes pullatus, Polychrus marmoratus y Chironius monticola). El ACP (iv) explicó el $62,3 \%$ de la variabilidad de los datos para los valores de uso de reptiles. El C1 $(40,7 \%)$ (uso directo) agrupó los valores de uso comercial $(0,96)$, ornamental $(0,92)$, artesanal y cacería $(0,77)$, con la especie Trachemys scripta como la más utilizada para la venta y Anolis ventrimaculatus, Thecadactylus rapicauda, Chironius monticola y Polychrus marmoratus como las más relacionadas con mitos y rituales. El C2 (21,6\%) (uso indirecto) congregó los valores de uso negativo $(0,64)$ y pedagógico $(0,59)$ (Tabla 3 ). Se destacó a la iguana (Iguana iguana) como la más usada para turismo, medicina y alimento (carne y huevos) y a la serpiente mataganado (Micrurus dumerilii) como de uso negativo, pedagógico (peligro que representa para comunidades locales) y artesanal (uso de la piel). 


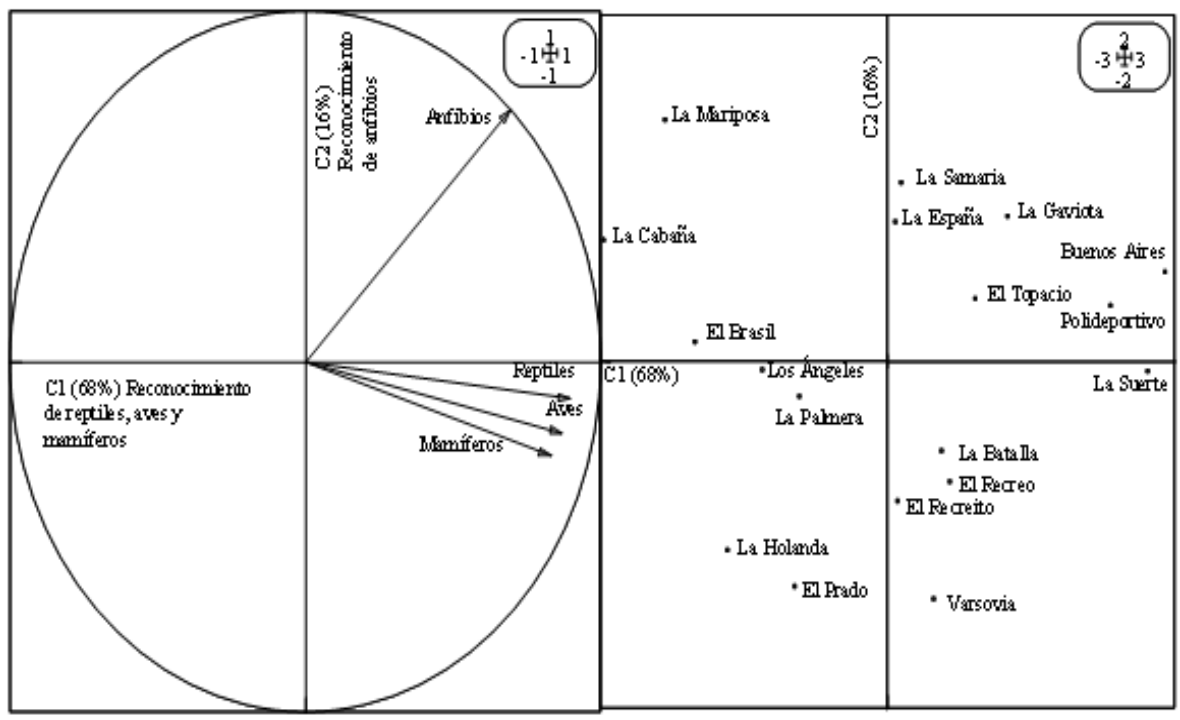

Fuente: los autores.

Figura 2. Círculos de correlaciones con los componentes 1 y 2 extraídos en el ACP de las variables relacionadas con el conocimiento de la fauna silvestre para los campesinos; proyección de los predios en los planos factoriales ( $\mathrm{C} 1$ y C2).

Tabla 3. Correlación de cada variable de uso con los componentes uno y dos extraídos en el ACP para mamíferos, aves y reptiles

\begin{tabular}{|c|c|c|c|c|c|c|}
\hline \multirow{2}{*}{$\begin{array}{c}\text { Grupos } \\
\text { Componentes } \% \\
\text { Variabilidad }\end{array}$} & \multicolumn{2}{|c|}{ Aves } & \multicolumn{2}{|c|}{ Mamíferos } & \multicolumn{2}{|c|}{ Reptiles } \\
\hline & C1 & $\mathrm{C} 2$ & C1 & $\mathrm{C} 2$ & $\mathrm{C}_{1}$ & $\mathrm{C} 2$ \\
\hline Alimento & 0,06 & 0,60 & 0,68 & 0,52 & 0,71 & $-0,54$ \\
\hline Medicina & $-0,18$ & 0,58 & 0,89 & $-0,39$ & 0,43 & $-0,66$ \\
\hline Comercial & 0,90 & $-0,13$ & 0,61 & 0,27 & 0,96 & 0,09 \\
\hline Artesanal & 0,00 & 0,00 & 0,74 & 0,38 & 0,77 & 0,53 \\
\hline Ornamental & 0,88 & $-0,18$ & 0,50 & 0,60 & 0,92 & 0,20 \\
\hline Investigación & 0,55 & 0,31 & 0,91 & $-0,33$ & 0,00 & 0,00 \\
\hline Cacería & 0,74 & 0,30 & 0,81 & 0,41 & 0,77 & 0,53 \\
\hline Observación & 0,39 & 0,30 & 0,49 & 0,62 & 0,65 & 0,01 \\
\hline Turismo & 0,64 & $-0,23$ & 0,78 & 0,15 & 0,69 & $-0,56$ \\
\hline Mitos y rituales & $-0,33$ & 0,27 & 0,89 & $-0,37$ & $-0,40$ & $-0,34$ \\
\hline Arte y literatura & 0,19 & 0,76 & 0,87 & $-0,26$ & 0,00 & 0,00 \\
\hline Pedagógico & $-0,13$ & 0,58 & 0,68 & $-0,47$ & 0,02 & 0,59 \\
\hline Negativo & $-0,03$ & 0,06 & $-0,40$ & 0,12 & $-0,35$ & 0,64 \\
\hline Benéfico & $-0,36$ & $-0,03$ & 0,92 & $-0,32$ & $-0,18$ & 0,26 \\
\hline
\end{tabular}

C1: Componente 1; C2: Componente 2.

$$
\text { Fuente: los autores. }
$$

Hábitats disponibles para aves, mamíferos, anfibios y reptiles en los usos del terreno 
El grupo que tuvo mayor presencia en los usos y coberturas del terreno fue el de las aves con valores más altos en frutales (47,5\%), platanal/bananal (45\%) y cafetales con sombrío (40\%). El segundo grupo fue el de mamíferos con alta presencia en los relictos de vegetación secundaria (40\%), cuerpos de agua $(27,5)$, maizales y guaduales $(27,5)$. En el caso de anfibios y reptiles se observó más altos valores en las gramíneas, caña panelera (31 y 35\%, respectivamente) y pastizales (25 y 32,5\%). Algunos cultivos como caña panelera y yuca presentaron los valores más bajos en la frecuencia de aparición de los grupos (Tabla 4).

Tabla 4. Percepciones de los campesinos de la presencia total en porcentaje de la fauna silvestre por coberturas y usos del terreno

\begin{tabular}{lccccc}
\hline \multirow{2}{*}{ Cobertura/ Uso del terreno } & \multicolumn{5}{c}{ Fauna silvestre (\%) } \\
\cline { 2 - 6 } & Aves & Mamíferos & Anfibios & Reptiles & Total \\
\hline Cacaotal & 34,1 & 22,6 & 23,3 & 20 & 100 \\
Cafetal con sombrio & 40 & 25 & 12,5 & 22,5 & 100 \\
Platanal/bananal & 45 & 22,5 & 20 & 12,5 & 100 \\
Yucal & 25 & 25 & 27,5 & 22,5 & 100 \\
Maizal & 45 & 27 & 17,5 & 10 & 100 \\
Cańa panelera & 17,5 & 16 & 31 & 35 & 100 \\
Frutales & 47,5 & 25 & 17,5 & 10 & 100 \\
Pastizal & 27,5 & 15 & 25 & 32,5 & 100 \\
Relicto vegetación secundaria & 22,5 & 40 & 20 & 17 & 100 \\
Cuerpos de agua & 25 & 27 & 22,5 & 25 & 100 \\
Guaduales & 35 & 27 & 17,5 & 20 & 100 \\
\hline
\end{tabular}

Fuente: los autores.

\section{DISCUSIÓN}

\section{Usos y coberturas del terreno y presencia de fauna silvestre}

Las coberturas y usos del terreno en la zona rural del municipio de Alcalá cambiaron drásticamente en la última década del siglo XX. La transformación del territorio generó una matriz de cobertura vegetal diversificada que al agregarse configura una malla de mosaicos que brindan refugio, alimento y corredores de desplazamiento para otros organismos del reino Animalia. La conservación en la memoria oral de sus nombres, usos y comportamientos, relación con las 
temporadas de lluvias, con las fases lunares, etc., es parte de la herencia cultural que transmiten los mayores a los jóvenes de la familia ampliada y del vecindario. El reconocimiento no solo implica la separación de la especie animal no humana, sino que también promueve la observación cuidadosa de las costumbres de otras formas de vida y de su interacción que al documentarse en un proceso de investigación-acción ayudaría a potencializar la conservación de la diversidad viviente.

La situación que se puede asociar como amigable para la presencia de aves, es la introducción de árboles frutales perennes (cítricos, aguacate, cacao, zapote, platanal/bananal,) que amplían la oferta de alimento insectil (Peraza et al., 2003), frutal, flores y néctar (Perfecto et al., 1996) y que brindan protección contra depredadores; situación contraria a lo que ocurre en los relictos de vegetación andina secundaria. En mamíferos los hábitats de mejor calidad fueron los relictos de vegetación secundaria; mientras que el pastizal ofreció pocos recursos alimenticios y por su consistencia herbácea los expone al riesgo de depredación (Guzmán, Lenis y Camargo, 2004). La presencia de anfibios se asoció más con coberturas hídricas y vegetación ripariana, debido a las condiciones fisiológicas que requieren (ciclo de vida acuático-terrestre, ectotermia, permeabilidad integumentaria) y a la alta dependencia de las condiciones ambientales favorables y a la alta sensibilidad a cambios en su hábitat por el impacto antrópico (RomeroMartínez et al., 2008). Las gramíneas ofrecieron espacios propicios para los reptiles (caña panelera, 35\%; pastizal, 32\%), ya que su condición herbácea opone menor resistencia a la reptación y la función de depredación. Asimismo, los registros pudieron estar relacionados con la facilidad de avistamiento para la visión mesoscópica en sitios abiertos y por ser los lugares que los agricultores frecuentan cotidianamente. Los resultados con relación al grupo ameritan profundizar en futuras investigaciones ya que van en contravía a los obtenidos por CarvajalCogollo y Urbina-Cardona (2008), quienes destacan a los pastizales del bosque seco tropical del Caribe colombiano como medio poco propicio para la presencia de herpetofauna debido a la reducción en el área disponible, pérdida de calidad del hábitat y aumento en la perturbación antropogénica.

El número de organismos reconocidos de fauna silvestre, 168 (103 de aves, 31 de mamíferos, 17 de anfibios, 17 de reptiles) casi duplica a lo realizado por las comunidades indígenas Embera-Katíos de la cuenca del río San Jorge, Córdoba, Colombia (88 especies: 29 de mamíferos, 25 de aves, 19 de reptiles y 15 de anfibios) (Racero et al., 2008), pero las dos indagaciones evidencian el valor de los saberes locales en la conservación de la vida. La hipótesis formulada confirmó la 
necesidad del encuentro sinérgico y de los repertorios culturales locales para potenciar las políticas públicas de conservación de la diversidad biológica y la asociación de la calidad de los hábitats para la fauna silvestre con los estilos de agricultura de la localidad (campesina, empresarial, combinaciones de ellas). La indagación puede contribuir a reorientar el enfoque de conservación desde arriba y como exclusiva responsabilidad de expertos. La orientación promueve la necesidad del abordaje desde el nivel de los actores de cada localidad agregados en mosaicos de vecindad para luego pasar a los niveles de microcuencas y cuencas territoriales.

\section{Aportes a los registros de fauna silvestre en el Eje Cafetero colombiano}

Colombia ha sido destacada como el país más diverso en aves del mundo con 1897 especies (Donegan et al., 2012), el cuarto en mamíferos con aproximadamente 479 (Chaves y Santamaría, 2006), el segundo en anfibios con 714 (Galeano et al., 2006) y el tercero en reptiles con 524 especies (Páez et al., 2006). La contribución de los expertos a complementar los registros nacionales de especies de aves, mamíferos, anfibios y reptiles con hallazgos en el Eje Cafetero colombiano ha sido importante, por ejemplo en aves Fajardoet al. (2009) encontraron 229 especies en parcelas con diversos usos del terreno en la cuenca del río La Vieja (localizada entre los departamentos de Quindío, Risaralda y Valle del Cauca), mientras que en Génova, departamento del Quindío, se registraron 98 especies en Páramos y Bosques Alto-andinos (Fundación Ecológica Reserva Las Mellizas, 2005). En el caso de los mamíferos, Castaño et al. (2003) registraron 124 especies en 29 localidades de 13 municipios en el departamento de Caldas, mientras que Orjuela y Jiménez (2004) presentaron indicios de nueve especies de mamíferos en bosques secos del departamento de Risaralda; entre tanto, en el mismo año, Sánchez, Sánchez-Palomino y Cadena (2004) identificaron 42 especies del mismo grupo en la Reserva Río Blanco, departamento de Caldas. Para anfibios Cadavid, Román-Valencia y Gómez (2005) capturaron 16 especies en seis estaciones situadas en los municipios de Armenia y La Tebaida en el departamento del Quindío.

El aporte realizado en este trabajo con la asociación de la dupla investigadorcampesino pudo cuantificar en las coberturas y usos del terreno de las fincas, con aproximadamente 200 ha (Tabla 1), 103 especies de aves $(5,4 \%$ del total encontrado en Colombia); 31 de mamíferos (6,4\%); 17 de anfibios (2,3\%) y 17 de reptiles (3,2\% del total nacional) (Tabla 2). La contribución también mostró el valor de la dupla en la interlocución al brindar información que permite ampliar los 
registros de una pequeña área del municipio de Alcalá, que cuenta con aproximadamente $61 \mathrm{~km}^{2}$, hecho que resalta y denota el valor de los hallazgos en la consolidación del conocimiento. Lo anterior se suma a otro esfuerzo llevado a cabo por Zúñiga et al. (2013) con el estudio de las percepciones de los agricultores sobre las lombrices de tierra, lo cual denota continuidad en la línea de investigación y resalta la importancia de incorporar la oralidad en los estudios de los expertos.

\section{Interlocutores locales como fuente de consolidación del conocimiento}

El papel que ha jugado la fauna silvestre en la estructuración del pensamiento sobre la naturaleza de los grupos sociales colombianos tanto en términos simbólicos como materiales (Baptiste et al., 2002), cambia de valor en la medida en que las presiones demográficas y el poder de los propietarios de la tierra aceleran la transformación de los hábitats, la sustitución de coberturas agrícolas y la ampliación de las coberturas construidas (Bulte y Horan, 2003).

Los valores de uso de la fauna silvestre dependen en gran parte de la cultura y de las preferencias de los sectores de la sociedad (Swift et al., 2004). Por ejemplo, los campesinos de la cuenca del río La Vieja usaron en cacería 52 de 76 especies silvestres (28 aves, 17 mamíferos y siete reptiles). El resultado se asemeja al documentado por González (2003b) en la Reserva Comunal Yanesha, Perú, donde comunidades nativas y colonos cazan 44 especies (23 aves, 18 mamíferos y tres reptiles); pero se diferenciaron del de indígenas y colonos de Imataca (Bolívar, Venezuela) que solo informaron del consumo de 17 especies (13 de mamíferos, tres aves y un reptil) (Bisbal, 1994), y de afroamericanos de la cuenca media del río Atrato (Chocó, Colombia) que mencionaron la caza de 20 especies (13 mamíferos, seis aves y un reptil) (Palacios, Rodríguez y Jiménez, 2008).

Entre las 25 especies de mamíferos de diferentes usos, los armadillos (Dasypus novemcinctus y Cabassous centralis) se calificaron como benéficos para la salud por el uso de la sangre como remedio para el asma; uso que también ha sido narrado por campesinos de la Serranía de Mamapacha en Boyacá, Colombia (García, Perico y Rocha, 2008). Los caparazones se emplean como recipientes para almacenar huevos y en la elaboración de instrumentos musicales (carrasca y lira), a diferencia de pobladores del Norte de Santander, Colombia, donde los utilizan como materas (Díaz y Moreno, 2003). 
Los campesinos percibieron 40 especies silvestres como negativas por causar daños en los cultivos (17 mamíferos, 14 aves y nueve reptiles). En la Amazonía oriental peruana los colonos nombraron 19 aves, 13 mamíferos y un reptil como perjudiciales para la agricultura (González, 2003a). Los periquitos y la cotorra frente roja fueron las especies más usadas en comercio, ornamento y cacería. De la misma manera las comunidades rurales de Norte de Santander, Colombia (Díaz y Moreno, 2003) y las Embera-Katíos de la cuenca del río San Jorge, Córdoba, Colombia (Racero et al., 2008) utilizan aves de la familia Psittacidae como ornamento y comercio. Alrededor de los reptiles se ha tejido toda clase de fantasías que generan temor en la comunidad (Castaño, 2002; Racero et al., 2008). Los campesinos de la cuenca del río La Vieja calificaron a la serpiente coral o mataganado como negativa por ser venenosa.

Partir de la proximidad, de lo familiarizado, de lo transmitido por los mayores, constituye una ruta que permitiría potenciar las estrategias convencionales de conservación de la diversidad biológica. Las fincas que han distribuido sus terrenos para la coexistencia de la naturaleza viva, de las culturas campesinas y de la cultura empresarial en la cuenca del río La Vieja se han convertido en conservatorios para la fauna silvestre que les proporciona espacio, refugio acogida, abrigo y alimento. Al compartir los espacios de la existencia campesina, las conductas animales se integran a las experiencias vividas por las personas locales sirviendo como beneficio o perjuicio; como anunciadoras de cambios lunares, de temporadas del clima, de desastres naturales; como acompañantes, como generadoras de afectos infantiles; o enriqueciendo los símbolos de la cultura.

\section{AGRADECIMIENTOS}

Los autores agradecen a los campesinos por el acompañamiento en la realización del trabajo. A la Universidad Tecnológica de Pereira y a Colciencias por financiar los proyectos "Evaluación del aporte de algunos servicios ambientales en fincas de la cuenca del río La Vieja, Colombia" y "Valoración de los bienes y servicios de la biodiversidad para el desarrollo sostenible de paisajes rurales colombianos: Complejo Ecorregional Andes del Norte", este último adscrito al Centro de Investigaciones y Estudios en Biodiversidad y Recursos Genéticos (CIEBREG). También a los compañeros del grupo Gestión en Agroecosistemas Tropicales Andinos - Planificación Socio-Ecológica del Paisaje (GATA-PSEP) por su respaldo y apoyo. 


\section{POTENCIAL CONFLICTO DE INTERESES}

El presente manuscrito enviado a la Revista Luna Azul no presenta conflicto de intereses porque es el resultado de un proyecto de investigación ejecutado por los responsables y por lo tanto declaramos que es un producto original.

\section{REFERENCIAS}

- Baptiste, L.; Hernández, S.; Polanco, R. y Quiceno, M. (2002). La fauna silvestre colombiana: una historia económica y social de un proceso de marginalización. En Ulloa, A.; Baptiste, L.; Campos, C.; Cayón, L.; Falchetti, A.; Guzmán, A.; Hernández, S.; Montoya, S.; Nates, G.; Orrantia, J.; Pinzón, C.; Polanco, R.; Quiceno, M.; Salcedo, M.; Tapi, L.; Tovar, P. y Turbay, S. (Eds.), Rostros culturales de la fauna. Las relaciones entre los humanos y los animales en el contexto colombiano (pp. 113-129). Bogotá: Instituto Colombiano de Antropología e Historia - Fundación Natura - McArthur Foundation.

- Bisbal, F. (1994). Consumo de fauna silvestre en la zona de Imataca, estado Bolívar, Venezuela. Interciencia, 19, 1-6.

- Bulte, E. y Horan, R. (2003). Habitat conservation, wildlife extraction and agricultural expansion. Journal of Environmental Economics and Management, 45, 109-127.

- Cadavid, J.G.; Román-Valencia, C. y Gómez, A.F. (2005). Composición y estructura de anfibios anuros en un transecto altitudinal de los Andes Centrales de Colombia. Revista Museo Argentino Ciencias Naturales, 7, 103-118.

- Caicedo, G. (1994). Atlas de Recursos Naturales del Valle del Cauca, (Tomo

2). Cali: Corporación Autónoma Regional del Valle, CVC.

- Carvajal-Cogollo, J.E. y Urbina-Cardona, J.N. (2008). Patrones de diversidad y composición de reptiles en fragmentos de bosque seco tropical en Córdoba, Colombia. Tropical Conservation Science, 1(4), 397-416. 
- Castaño, J.H.; Muñoz-Saba, Y.; Botero, J.E. y Vélez, J.H. (2003). Mamíferos del departamento de Caldas - Colombia. Biota Colombiana, 4(2), 247-259.

- Castaño, O. (2002). Libro rojo de reptiles de Colombia. Serie Libros Rojos de Especies Amenazadas de Colombia. Bogotá: Instituto de Ciencias NaturalesUniversidad Nacional de Colombia, Ministerio del Medio Ambiente, Conservación Internacional-Colombia.

- Charnley, S.; Paige, A. y Jones, E.T. (2007). Integrating traditional and local ecological knowledge into forest biodiversity conservation in the Pacific Northwest. Forest Ecology and Management, 246, 14-28.

- Chaves, M.E. y Santamaría, M. (Eds.). (2006). Informe Nacional sobre el Avance en el Conocimiento y la Información de la Biodiversidad 1998-2004, (2 Tomos). Bogotá D.C.: Instituto de Investigación en Recursos Biológicos Alexander von Humboldt.

- Daniel, W. (1996). Bioestadística bases para el análisis de las ciencias de la salud. México: Editorial Limusa - Grupo Noriega Editores.

- De Albuquerque, C.A. y De Albuquerque, U.P. (2005). Local perceptions towards biological conservation in the community of Vila Velha, Pernambuco, Brazil. Interciencia, 30(8), 460-465.

- Deslauriers, J. (1991). Investigación cualitativa: Guía práctica. Versión al español y edición al cuidado de Gómez, M.Á. Universidad Tecnológica de Pereira, Risaralda, Colombia. Junio de 1999 (Primera versión).

- Díaz, L. y Moreno, F. (2003). Percepción del uso de la fauna silvestre y estrategias de conservación predial con comunidades rurales. Gestión $\boldsymbol{y}$ Ambiente, 6(2), 61-70.

- Donegan, T.; Quevedo, A.; Salaman, P. y McMullan, M. (2012). Revision of the status of bird species occurring or reported in Colombia 2012. Conservación Colombiana, 17, 4-14. 
- Durand, L. (2008). De las percepciones a las perspectivas ambientales: Una reflexión teórica sobre la antropología y la temática ambiental. Revista Nueva Antropología, 21(68), 75-87.

- Emmons, J. (1991). Neotropical rainforest mammal: a field guide. USA: The University of Chicago Press.

- Fajardo D.; Johnston-González, L.N.; Chará, J. y Murgueitio, E. (2009). Influencia de sistemas silvopastoriles en la diversidad de aves en la cuenca del río La Vieja, Colombia.Recursos Naturales y Ambiente, 58, 9-16.

- Feijoo, A.; Carvajal, A.F.; Zúñiga, M.C.; Quintero, H. y Fragoso, C. (2011). Diversity and abundance of earthworms in land use systems in centralwestern Colombia. Pedobiologia, 54S, S69-S75.

• Feijoo, A.; Zúñiga, M.C.; Quintero, H.; Carvajal, A. y Ortiz, D. (2010). Patrones de asociación entre variables del suelo y usos del terreno en la cuenca del río La Vieja, Colombia. Acta Zoológica Mexicana, 26, n.spe. 2, 151-164.

- Feijoo, A.; Zúñiga, M.C.; Quintero, H. y Lavelle, P. (2007). Relaciones entre el uso de la tierra y las comunidades de lombrices en la cuenca del río La Vieja, Colombia. Pastos y Forrajes, 30(2), 235-249.

- Fundación Ecológica Reserva Las Mellizas. (2005). Áreas de importancia para la conservación de las aves AICAS - COLOMBIA. Enriquecimiento del inventario de avifauna del AICA paramos y bosques altoandinos de Génova Quindío, y procesos de educación y sensibilización ambiental en torno a los ecosistemas de alta montaña. Bogotá: Instituto de Investigación de Recursos Biológicos Alexander von Humboldt.

- Galeano, S.P.; Urbina, J.C.; Gutiérrez-C., P.D.A.; Rivera-C., M. y Páez, V.P. (2006). Los anfibios de Colombia, diversidad y estado del conocimiento. En Chaves, M.E. y Santamaría, M. (Eds.), Informe nacional sobre el avance en el conocimiento y la información de la biodiversidad 1998-2004, (Tomo II) (pp. 106-118). Bogotá: Instituto de Investigaciones Biológicas Alexander von Humboldt.

- García, G.; Perico, D. y Rocha, C. (2008). Uso de fauna silvestre en los alrededores de la Serranía de Mamapacha (Boyacá, Colombia). Recuperado de http://www.lablaa.org/blaavirtual/geografia/congresoparamo/uso-de-fauna.pdf 
- González, J. (2003a). Análisis preliminar de los daños causados por las aves silvestres a la agricultura en la amazonía oriental del Perú. En Polanco, R. (Ed.), Manejo de fauna silvestre en Amazonía y Latinoamérica. V Congreso Internacional (pp. 370-380). Bogotá: CITES - Fundación Natura, Colombia.

- González, J. (2003b). Patrones generales de caza y pesca en comunidades nativas y asentamiento de colonos aledaños a la Reserva Comunal Yanesha, Pasco, Perú. En Polanco, R. (Ed.), Manejo de fauna silvestre en Amazonía y Latinoamérica. V Congreso Internacional (pp. 89-102). Bogotá: CITES Fundación Natura, Colombia.

- Guber, R. (1994). El salvaje metropolitano. Reconstrucción del conocimiento social en el trabajo de campo. Buenos Aires, Argentina: Paidós.

- Guzmán, A.; Lenis, A. y Camargo, S. (2004). Importancia de los rastros para la caracterización del uso de hábitat de mamíferos medianos y grandes en el bosque Los Mangos (Puerto López, Meta, Colombia). Acta Biológica Colombiana, 9(1), 11-22.

- Hilty, S. y Brown, W. (1986). A Guide of the Birds of Colombia. USA: Princeton University Press.

- Hobbs, R.J.; Arico, S.; Aronson, J.; Baron, J.S.; Bridgewater, P.; Cramer, V.A.; Epstein, P.; Ewel, J.J.; Klink, C.A.; Lugo, A.E.; Norton, D.; Ojima, D.; Richardson, D.M.; Sanderson, E.W.; Valladares, F.; Vilà, M.; Zamora, R. y Zobel, M. (2006). Novel ecosystems: theoretical and management aspects of the new ecological world order. Global Ecology and Biogeography, 15, 1-7.

- Ingold, T. (2000). The Perception of environment. Essays of livelihood, dwelling and skill. London: Routledge.

- Marshall, E.; West, T. y Kleijn, D. (2006). Impacts of an agri-environment field margin prescription on the flora and fauna of arable farmland in different landscapes. Agriculture, Ecosystems and Environment, 113, 36-44.

- McNeely, J.A.; Miller, K.R.; Reid, W.R.; Mittermeier, R.A. y Werner, T.B. (1990). Conserving the world's biological diversity. En Conserving the world biological diversity(pp. 25-34). Washington, DC: IUNC. 
- MEA. (2005a). Ecosystems and human well-being: current state and trends: Findings of the Conditions and Trends Working Group. En Hassan, R.; Scholes, R. y Ash, N. (Eds.),Millennium Ecosystem Assessment (MA). Washington, DC: Island Press.

- MEA. (2005b). Ecosystems and human well-being: biodiversity synthesis. Washington, DC: Island Press.

- Millington, J.; Walters, M.; Matonis, M. y Liu, J. (2010). Effects of local and regional landscape characteristics on wildlife distribution across managed forests. Forest Ecology and Management, 259, 1102-1110.

- Nyeko, P.; Edwards-Jones, G.; Day, R. y Raussen, K.T. (2002). Farmers' knowledge and perceptions of pests in agroforestry with particular reference to Alnus species in Kabale district, Uganda. Crop Protection, 21, 929-941.

- Orjuela, O.J. y Jiménez, G. (2004). Estudio de la abundancia relativa para mamíferos en diferentes tipos de coberturas y carretera, finca hacienda Cristales, área Cerritos - La Virginia, municipio de Pereira, departamento de Risaralda Colombia. Universitas Scientiarum, 9, 87-96.

- Páez, V.P.; Arredondo, J.C.; López, C.; Martínez, L.M.; Molina, C. y Restrepo, A. (2006). Los reptiles de Colombia, diversidad y estado de conocimiento. En Chaves, M.E. y Santamaría, M. (Eds.), Informe sobre el avance en el conocimiento y la información de la biodiversidad 1998-2004, (Tomo II) (pp. 118-130). Bogotá D.C., Colombia: Instituto de Investigación de Recursos Biológicos Alexander von Humboldt.

- Palacios, Y.; Rodríguez, A. y Jiménez, A. (2008). Aprovechamiento de los recursos naturales por parte de la comunidad local en la cuenca media del río Atrato, Chocó, Colombia. Revista Institucional Universidad Tecnológica del Chocó: Investigación, Biodiversidad y Desarrollo, 27(2), 175-85.

- Peraza, C.; Cifuentes, Y.; Alayon, Y. y Clavijo, C. (2003). Adiciones a la avifauna de un cafetal con sombrío en la Mesa de los Santos (Santander, Colombia). Universitas Scientiarum, 9, 19-32. 
- Perfecto, I.; Rice, R.; Greenberg, R. y Van Der Voort, M. (1996). Shade coffee: a disappearing refuge for biodiversity. BioScience, 46(8), 598-608.

- Racero, J.; Vidal, C.; Ruiz, O. y Ballesteros, J. (2008). Percepción y patrones de uso de la fauna silvestre por las comunidades indígenas Embera-Katíos en la cuenca del río San Jorge, zona amortiguadora del PNN-Paramillo. Revista de Estudios Sociales, 31, 118-131.

- Romero-Martínez, H.J.; Vidal-Pastrana, C.; Lynch, J. y Dueñas, P. (2008). Estudio preliminar de la fauna amphibia en el cerro Murrucucú, Parque Natural Nacional Paramillo y zona amortiguadora, Tierralta, Córdoba, Colombia. Caldasia, 30(1), 209-229.

- Ruiz-Cobo, D.H.; Feijoo, A. y Rodríguez, C. (2010). Soil macro-invertebrate communities in different land use systems in the Otún River Valley, Colombia. Acta Zoológica Mexicana, 26, n.spe. 2, 165-178.

- Sánchez, F.; Sánchez-Palomino, P. y Cadena, A. (2004). Inventario de mamíferos en un bosque de los Andes centrales de Colombia. Caldasia, 26(1), 291-309.

- Sheil, D. y Lawrence, A. (2004). Tropical biologists, local people and conservation: new opportunities for collaboration. Trends Ecology and Evolution, 19, 634-638.

- Sheil, D.; Puri, R.; Basuki, I.; Heist, M.; Wan, M.; Liswanti, N.; Rukmiyati, I.; Sardjono, M.; Samsoedin, I.; Sidiyasa, K.; Permana, E.; Mangopo, E.; Gatzweiler, F.; Johnson, B. y Wijaya, A. (2003). Exploring biological diversity, environment and local people's perspectives in forest landscapes: methods for a multidisciplinary landscape assessment. Indonesia: CIFOR.

- Sheil, D.; Puri, R.; Wan, M.; Basuki, I.; Van Heist, M.; Liswanti, N.; Rumiyati, N.; Rachmatika, I. y Samsoedin, I. (2006). Recognizing Local People's Priorities for Tropical Forest Biodiversity. Ambio, 35, 17-24.

- Swift, M.; Izac, M. y Noordwijk, M. (2004). Biodiversity and ecosystem services in agricultural landscapes-are we asking the right questions? Agriculture, Ecosystems and Environment, 104, 113-134. 
- Tengberg, A.; Fredholm, S.; Eliasson, I.; Knez, I.; Saltzman, K. y Wetterberg, O. (2012). Cultural ecosystem services provided by landscapes: Assessment of heritage values and identity. Ecosystem Services, 2, 14-26.

- Thioulouse, J.; Chessel, D.; Dolédec, S. y Olivier, J. (1997). ADE4 Multivariate analysis and graphical display software. Statistical Computation, 7, 2-15.

- Tobasura, I. (1996). Una visión Integral de la Biodiversidad en Colombia. Revista Luna Azul, 2. Manizales: Universidad de Caldas. Recuperado de http://lunazul.ucaldas.edu.co/downloads/cd41ee01Revista2_4.pdf

- Tuan, Y.F. (1974). Topophilia, A Study of Environmental Perception, Attitudes, and Values. Englewood Cliffs (NJ): Prentice-Hall.

- Vargas, CH.D. (1984). Análisis de componentes principales. INIA, Mesa de control (Estadística y Cálculo). Oficinas Centrales. México.

- Velásquez, E.; Lavelle, P.; Barrios, P.; Joffre, R. y Reversat, F. (2005). Evaluating soil quality in tropical agroecosystems of Colombia using NIRS. Soil Biology \& Biochemistry, 37, 889-898.

- Zúñiga, M.C.; Feijoo, A. y Quintero, H. (2003). Trayectoria de los sistemas campesinos de cría en un área del piedemonte de Alcalá, Valle del Cauca. Scientia et Technica, 9, 81-86.

- Zúñiga, M.C.; Feijoo, A. y Quintero, H. (2004). Diseño de una propuesta metodológica para interpretar el huerto habitacional en un área del Valle del Cauca. Scientia et Technica, 10, 291-296.

• Zúñiga, M.C.; Feijoo, A.; Quintero, H.; Aldana, N. y Carvajal, A. (2013). Farmers' perception of the role played by earthworms in soil life. Applied Soil Ecology, 69, 61-68.

1. Magíster en Sociedades Rurales. Grupo Gestión en Agroecosistemas Tropicales Andinos, Facultad de Ciencias Ambientales, Universidad Tecnológica de Pereira. Pereira, Colombia.narlyj@utp.edu.co. ORCID: 0000-0001-6439-1037 
2. Magíster en Ecotecnología. Universidad Nacional de General Sarmiento, Área de Biología y Bioinformática, Instituto de Ciencias. Buenos Aires, Argentina.monidipo@utp.edu.co. ORCID: 0000-0003-4926-5184

3. Doctor en Ciencias Agrarias. Coordinador Grupo Gestión en Agroecosistemas Tropicales Andinos; profesor Facultad de Ciencias Ambientales, Universidad Tecnológica de Pereira. Pereira, Colombia. afeijoo@utp.edu.co. ORCID: 00000002-5226-5233.

4. Ingeniero Agrónomo. Profesor Pensionado, Universidad Nacional de Colombia, sede Palmira. Palmira, Colombia. hquinterov68@hotmail.com. ORCID: 0000-00034548-7169

Para citar este artículo: Aldana-Mejía, N.J.; Díaz-Porres, M.; Feijoo-Martínez, A. y Quintero, H. (2016). Percepciones y reconocimiento local de fauna silvestre, municipio de Alcalá, departamento del Valle del Cauca, Colombia. Revista Luna Azul, 43, 56-81. Recuperado de http://200.21.104.25/lunazul/index.php?option=com_content\&view=article\&id=193 